\title{
Pneumocystis Pneumonia after Kidney Transplantation in Children
}

\section{Soojin Hwang, M.D., Ph.D. Jiwon Jung, M.D. Joo Hoon Lee, M.D., Ph.D. Young Seo Park, M.D., Ph.D. \\ Department of Pediatrics, Asan Medical Center Children's Hospital, University of Ulsan College of \\ Medicine, Seoul, Korea}

Corresponding author:

Young Seo Park, M.D., Ph.D.

Department of Pediatrics, Asan Medical Center Children's Hospital, University of Ulsan College of Medicine, 88 Olympic-ro 43-gil, Songpa-gu, Seoul 05505, Korea

Tel: +82-2-3010-3376

Fax: +82-2-473-3725

E-mail:yspark@amc.seoul.kr

Received: 1 March 2020

Revised: 19 March 2020

Accepted: 25 March 2020
Pneumocystis pneumonia (PCP) is a rare disease in healthy people but a potentially fatal opportunistic infection by Pneumocystis jirovecii in immunocompromised patients with organ transplantation. We present three cases of PCP after kidney transplantation in pediatric patients. First case was a 4-year-old boy diagnosed with Denys-Drash syndrome and received living-donor kidney transplantation from his mother at age of 1 . Second case was a 19-year-old male, with polycystic kidney disease, who received kidney transplantation from his mother at the age of 18. Third case was a 19-year-old female with chronic kidney disease of unknown etiology, who received kidney transplantation from her father at age of 15 . These three patients who were on immunosuppressive therapy and completed of routine PCP prophylaxis for 6 months had presented with cough and dyspnea more than 1 year after transplantation. Chest $x$-ray all showed diffuse haziness of both lung fields, and bronchoalveolar lavage from bronchoscopy revealed Pneumocystis jirovecii infection. All patients showed clinical resolution with intravenous trimethoprim-sulfamethoxazole (TMP-SMX) therapy for at least 3 weeks and had continued secondary prophylaxis for another $6-12$ months. This report suggests that clinicians should have suspicion for the possibilities of opportunistic infection such as PCP after kidney transplantation in children.

Key words: Pneumocystis pneumonia, Kidney transplantation, Children

\section{Introduction}

Kidney transplantation has become the treatment of choice for children with end-stage renal disease. Kidney transplantation has the potential for normal growth and development with fewer complications ${ }^{1)}$. However, transplant recipients require lifelong maintenance immunosuppression. In kidney transplant recipients, opportunistic infections are causes of morbidity and mortality. Risk factors which affect the incidence and severity of opportunistic infections after kidney transplantation include transmission in graft, recipient immunity, immunosuppression medications, and availability of prophylaxis ${ }^{2)}$.

Pneumocystis pneumonia (PCP) is known to complicate $0.6-14 \%$ of patients with renal transplant recipients with a mortality rate of about $33 \%$ in the absence of prophylaxis ${ }^{3,4}$. The risk for infection is highest in first 6 months after transplantation due to intensified immunosuppressive therapy ${ }^{5}$. Oral trimethoprim-sulfamethoxazole (TMP-SMX) is the most effective agent for 
both treatment and prophylaxis of PCP. Therefore, routine PCP prophylaxis with TMP-SMX is recommended for at least 6 months after kidney transplantation by many centers, including our center ${ }^{6,7)}$. The incidence of PCP in renal transplant recipients has gradually decreased to $0.4-7 \%$ with the use of prophylaxis ${ }^{2,8)}$. Herein, we report three cases of pediatric kidney transplant recipients who developed late-onset PCP after the first year of transplantation.

\section{Case report}

First case was a 4-year-old boy diagnosed with DenysDrash syndrome and received living-donor kidney transplantation from his mother at 1 year old. To prevent acute rejection, we used basiliximab as induction immunosuppressant during the perioperative period. He presented with sore throat, cough and chest pain 3 years after transplantation while taking deflazacort ( $1 \mathrm{mg} / \mathrm{kg} /$ day $)$, tacrolimus $(0.16 \mathrm{mg} / \mathrm{kg} /$ day, a mean trough level of $10 \mathrm{ng} / \mathrm{mL})$, and mycophenolic acid (50 mg/kg/day) as maintenance therapy. The initial vital signs were as follows: blood pressure, 103/64 $\mathrm{mmHg}$; heart rate, 144 beats per minute; respiratory rate, 28 breaths per minute; temperature, $37.5^{\circ} \mathrm{C}$; Oxygen saturation, $96 \%$ on room air. His breathing sounds were coarse, and wheezing were present in both lung fields. Initial laboratory evaluation of complete blood count showed a white blood cell count of $8.7 \times 10^{9} / \mathrm{L}$ with $73.9 \%$ neutrophils, $15.3 \%$ lymphocytes, and $10.0 \%$ monocytes, hemoglobin $7.8 \mathrm{~g} / \mathrm{dL}$, and platelets $485,000 / \mathrm{mm}^{3}$. The C-reactive protein level was $0.12 \mathrm{mg} / \mathrm{dL}$ (normal $<0.6 \mathrm{mg} / \mathrm{dL}$ ). Venous blood gas analysis at room air yielded the following values: $\mathrm{pH}, 7.41$;
$\mathrm{PCO}_{2}, 46 \mathrm{mmHg} ; \mathrm{PO}_{2}, 37 \mathrm{mmHg}$; and $\mathrm{HCO}_{3}, 29.2 \mathrm{mEq} / \mathrm{L}$. The serum creatinine level was $0.85 \mathrm{mg} / \mathrm{dL}$. Initial plain chest radiography revealed bilateral haziness in both lung fields, suggestive of atypical pneumonia (Fig. 1A). We performed bronchoscopy and found positive polymerase chain reaction (PCR) result for Pneumocystis jirovecii from bronchoalveolar lavage. We started intravenous TMP-SMX and discontinued mycophenolic acid. While hospitalized, he showed substernal retraction and needed nasal cannula oxygen of $4 \mathrm{~L} / \mathrm{min}$. After 10 days of treatment, his cough and dyspnea resolved, and then supplemental oxygen was not required. He was treated with intravenous TMP-SMX (15 mg/kg/day TMP-75 mg/kg/day SMX) for total 3 weeks and continued PCP prophylaxis with oral TMP-SMX (6 $\mathrm{mg} / \mathrm{kg} /$ day TMP-30 mg/kg/day SMX) for another 6 months without recurrence. Currently, he is taking deflazacort, mycophenolic acid, and changed from tacrolimus to cyclosporine due to chronic diarrhea and failure to thrive.

Second case was a 19 -year-old male, with polycystic kidney disease, who received kidney transplantation from his mother at the age of 18 and basiliximab as induction therapy. One year after transplantation, he presented with symptoms of fever, cough, and tachypnea while taking deflazacort $(0.12 \mathrm{mg} / \mathrm{kg} /$ day $)$, tacrolimus $(0.16 \mathrm{mg} / \mathrm{kg} / \mathrm{day}$, a mean trough level of $5 \mathrm{ng} / \mathrm{mL}$ ), and mycophenolic acid (14.4 mg/kg/day). The patient was transferred from a local hospital, where he progressed to hypoxic respiratory failure and septic shock requiring endotracheal intubation and intravenous vasopressor within 24 hours of symptom onset. On arrival at our hospital, he was hospitalized to the pediatric intensive care unit and initial vital signs were as follows: blood pressure, 113/85 mmHg; heart rate, 108 beats per mi-

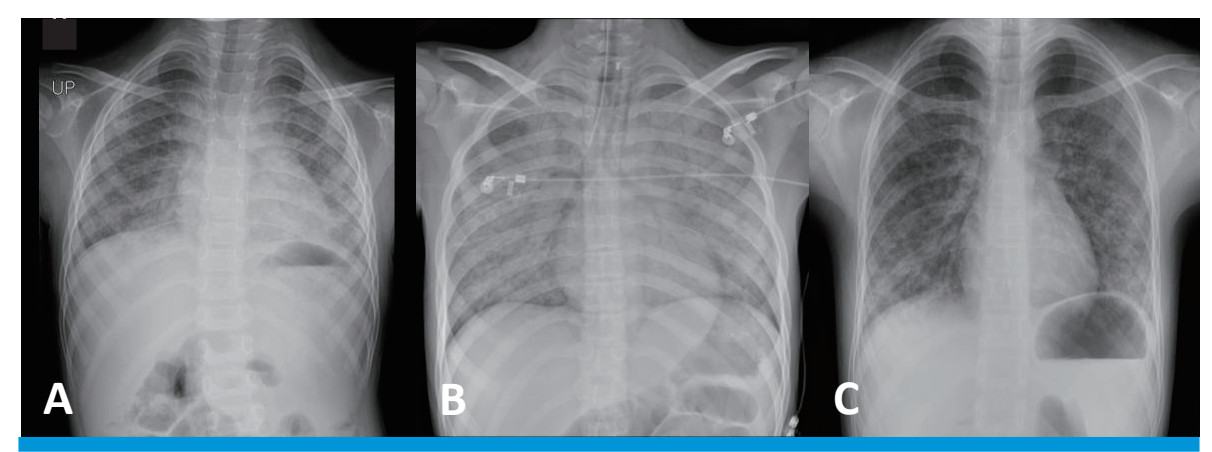

Fig. 1. Chest radiography. Chest radiography of $(A)$ the first patient showing bilateral haziness of lung fields, (B) the second patient showing diffuse ground glass opacities of both lungs, and (C) the third patient showing patchy ground glass opacities of both lungs. 
nute; respiratory rate, 27 breaths per minute; temperature, $36.6^{\circ} \mathrm{C}$; Oxygen saturation, $97 \%$ on pressure control ventilator with $\mathrm{FiO}_{2}$ of $60 \%$, positive end-expiratory pressure of $7 \mathrm{~cm} \mathrm{H}_{2} \mathrm{O}$, peak inspiratory pressure of $17 \mathrm{~cm} \mathrm{H}_{2} \mathrm{O}$ and a respiratory rate of 13 per minute. Coarse crackles were present from bilateral lung fields. Laboratory evaluation showed a white blood cell count of $7.7 \times 10^{9} / \mathrm{L}$ with $78 \%$ neutrophils, $15.8 \%$ lymphocytes, and $4.8 \%$ monocytes, hemoglobin $10.0 \mathrm{~g} / \mathrm{dL}$, and platelets $193,000 / \mathrm{mm}^{3}$. The C-reactive protein level was elevated at $14.16 \mathrm{mg} / \mathrm{dL}$. His arterial blood gas analysis showed the following values: $\mathrm{pH}, 7.41 ; \mathrm{PCO}_{2}, 35$ mmHg; $\mathrm{PO}_{2}, 77 \mathrm{mmHg}$; and $\mathrm{HCO}_{3}, 22.2 \mathrm{mEq} / \mathrm{L}$ on $\mathrm{FiO}_{2}$ of $60 \%$. Serum creatinine level was mildly elevated at 1.59 $\mathrm{mg} / \mathrm{dL}$. Plain chest radiography and computed tomography (CT) of lung showed diffuse ground glass opacities, suggesting interstitial pneumonia (Fig. $1 \mathrm{~B}$ and $2 \mathrm{~A}, \mathrm{~B}$ ). On the third day of hospital stay, bronchoalveolar lavage was done and positive results for Pneumocystis jirovecii were found from immunohistochemistry and PCR. He was initially treated with intravenous cefepime, azithromycin, and
TMP-SMX for both communityacquired bacterial pneumonia or possibly atypical pneumonia and discontinued mycophenolic acid. On the second day of hospitalization, he was extubated and changed to high flow nasal cannula. Finally, he had improved to breathe at room air on the ninth day. He completed a total 5 weeks of intravenous and oral TMP-SMX (15 mg/kg/day TMP-75 mg/kg/day SMX) treatment and started PCP prophylaxis with oral TMP-SMX (80 mg/day TMP-400 mg/day SMX) for another 12 months. And then, he is on maintenance immunosuppressive therapy with deflazacort and tacrolimus.

Third case was a 19-year-old female with chronic kidney disease of unknown etiology. She received kidney transplantation from her father at the age of 15 and had induction immunosuppression with basiliximab. However, acute cellular rejection presented at two years after transplantation and intensive immunosuppressive therapy was administered with double strength steroid for a while. At three years after transplantation, she developed dyspnea while taking deflazacort $(0.61 \mathrm{mg} / \mathrm{kg} /$ day $)$, tacrolimus $(0.1 \mathrm{mg} /$

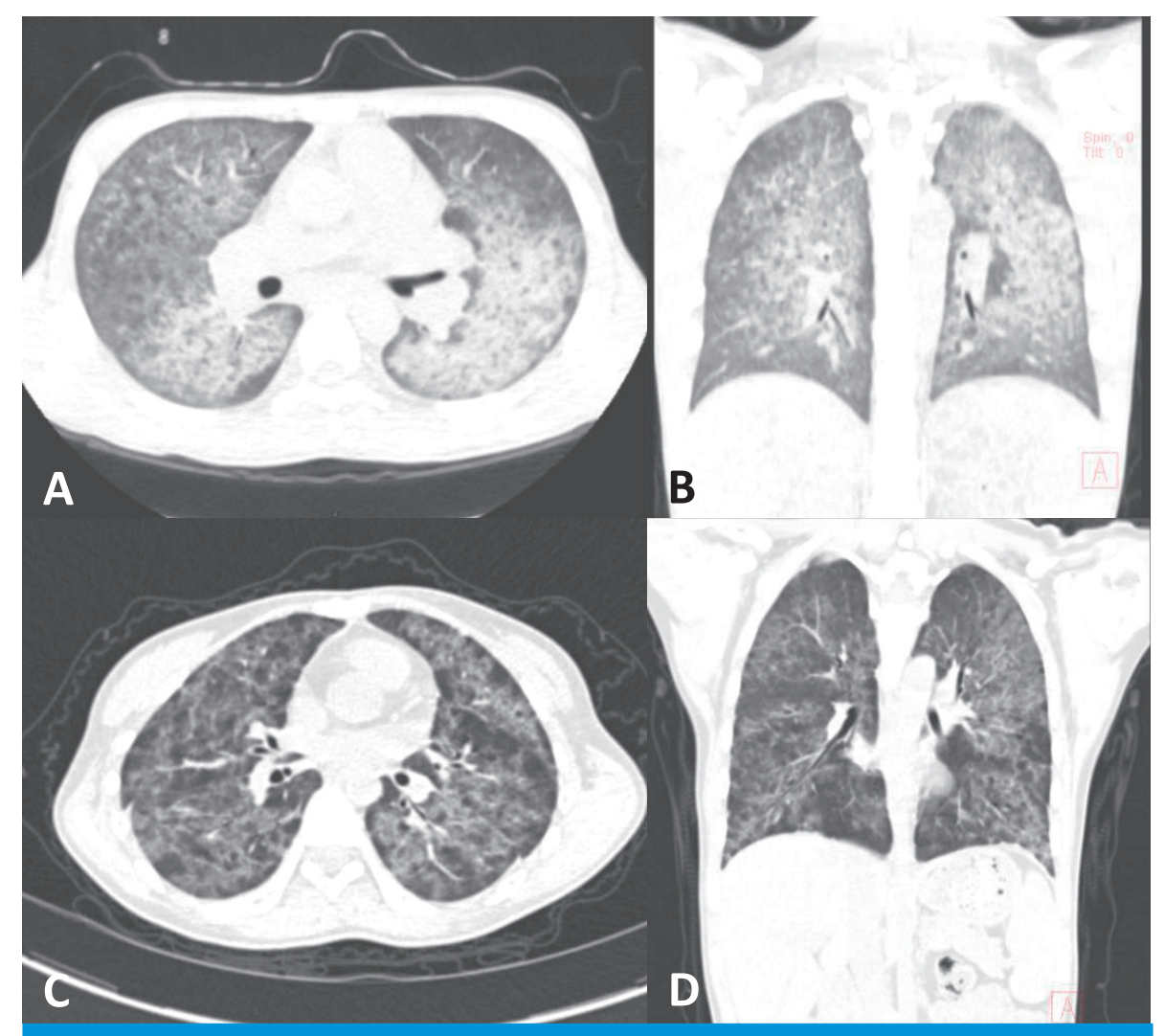

Fig. 2. Computed tomography (CT). (A) and (B): chest $C T$ of the second patient shows diffuse ground glass opacities of both lungs. $(C)$ and $(D)$ : chest $C T$ of the third patient shows diffuse patchy opacities of both lung fields. 
$\mathrm{kg} /$ day, a mean trough level of $7 \mathrm{ng} / \mathrm{mL}$ ), and mycophenolic acid (12.82 mg/kg/day). The initial vital signs were as follows: blood pressure, $107 / 75 \mathrm{mmHg}$; heart rate, 93 beats per minute; respiratory rate, 24 breaths per minute; temperature, $36.3^{\circ} \mathrm{C}$; Oxygen saturation, $99 \%$ on room air. Auscultation revealed wheezing from bilateral lung fields. Initial laboratory evaluation showed a white blood cell count of 5.0x $10^{9} / \mathrm{L}$ with $69.6 \%$ neutrophils, $17.1 \%$ lymphocytes, and 9.3 $\%$ monocytes, hemoglobin $8.4 \mathrm{~g} / \mathrm{dL}$, and platelets 551,000/ $\mathrm{mm}^{3}$. The C-reactive protein level was mildly elevated at $2.3 \mathrm{mg} / \mathrm{dL}$. Venous blood gas analysis at room air showed the following values: $\mathrm{pH}, 7.39$; $\mathrm{PCO}_{2}, 45$ mmHg; $\mathrm{PO}_{2}, 33$ $\mathrm{mmHg}$; and $\mathrm{HCO}_{3}, 27.0 \mathrm{mEq} / \mathrm{L}$. The serum creatinine level was mildly elevated at $1.83 \mathrm{mg} / \mathrm{dL}$. Plain chest radiography and CT showed diffuse patchy ground glass opacities in both lung fields, suggesting atypical or viral pneumonia (Fig. 1C and 2C, D). Bronchoscopy was done on the third day of hospital stay, and PCR for Pneumocystis jirovecii from a bronchoalveolar lavage was positive. On the impression of atypical pneumonia, she was started treatment with levofloxacin and TMP-SMX. She required oxygen therapy via nasal cannula of 1-3 L/min for 10 days of hospital stay, and gradually improved to breathe at room air afterwards. She completed TMP-SMX treatment for total 3 weeks and continued another 6 months. However, she was later diagnosed and treated for post-transplantation lymphoprolife- rative disorder (PTLD) 4 years after transplantation. Starting chemotherapy for PTLD, we changed immunosuppressive regimen from tacrolimus and mycophenolic acid to sirolimus $(0.15 \mathrm{mg} / \mathrm{kg} /$ day $)$ and prednisolone $(0.25 \mathrm{mg} / \mathrm{kg} /$ day $)$. Two more recurrent PCP occurred in 5 and 7 years posttransplantation. At each time of recurrence, she was treated with intravenous and oral TMP-SMX (15 mg/kg/day TMP$75 \mathrm{mg} / \mathrm{kg} /$ day SMX) for 3 weeks and continued additional prophylaxis with oral TMP-SMX (6 mg/kg/day TMP-30 $\mathrm{mg} / \mathrm{kg} /$ day SMX) for at least 6 months, respectively. Still, she is on maintenance immunosuppression with prednisolone and sirolimus. Clinical characteristics of three cases are summarized in Table 1.

\section{Discussion}

It is well known that PCP resulted in a worse clinical course and could be a cause of morbidity and mortality in renal transplant recipients receiving immunosuppressive therapy ${ }^{4)}$. Previous studies reported the incidence of PCP in adult renal transplant recipients were $0.6-14 \%^{2,3,8)}$, but there is no incidence data for children. This study reported three patients who had PCP after pediatric kidney transplantation which occurred after the completion of primary prophylaxis for PCP, with an incidence rate of $2.08 \%$ in our

Table 1. Clinical Characteristics of the Patients

\begin{tabular}{|c|c|c|c|}
\hline Characteristic & Patient 1 & Patient 2 & Patient 3 \\
\hline Age at diagnosis (yr) & 4 & 19 & 19 \\
\hline Gender & Male & Male & Female \\
\hline Underlying etiology for renal disease & Denys - Drash syndrome & Polycystic kidney disease & Unknown \\
\hline Type of kidney transplantation & Living-donor (mother) & Living-donor (mother) & Living-donor (father) \\
\hline Immunosuppressant drugs at diagnosis & $\begin{array}{c}\text { Deflazacort } \\
\text { Tacrolimus } \\
\text { Mycophenolic acid }\end{array}$ & $\begin{array}{c}\text { Deflazacort } \\
\text { Tacrolimus } \\
\text { Mycophenolic acid }\end{array}$ & $\begin{array}{c}\text { Deflazacort } \\
\text { Tacrolimus } \\
\text { Mycophenolic acid }\end{array}$ \\
\hline Primary prophylaxis duration after transplantation (mo) & 6 & 6 & 6 \\
\hline Time between PCP and transplantation (yr) & 3 & 1 & 3 \\
\hline Symptoms & $\begin{array}{l}\text { Sore throat } \\
\text { Cough } \\
\text { Chest pain }\end{array}$ & $\begin{array}{c}\text { Fever } \\
\text { Cough } \\
\text { Tachypnea }\end{array}$ & Dyspnea \\
\hline P.jirovecii PCR from bronchoalveolar lavage & Positive & Positive & Positive \\
\hline Respiratory support & Nasal cannula & Ventilator & Nasal cannula \\
\hline Treatment duration with TMP-SMX (wk) & 3 & 5 & 3 \\
\hline Secondary prophylaxis duration after treatment (mo) & 6 & 12 & 6 \\
\hline Recurrence & No & No & Yes* $^{*}$ \\
\hline
\end{tabular}

*The third patient was converted to prednisolone and sirolimus because of post-transplantation lymphoproliferative disorder (PTLD) in 4 years after kidney transplantation. She had unusual recurrences of PCP at 5 and 7 years after kidney transplantation. 
center from 1995 to 2019.

Renal transplant recipients receive intensive immunosuppressive therapy after transplantation, with combination of steroid and calcineurin inhibitor and antimetabolite agents. Therefore, PCP after renal transplantation usually occurred within 6 months and most patients were diagnosed within 1 year because of intense immunosuppression, but lateonset beyond 12 months are rare ${ }^{5,9)}$. For this reason, renal transplant patients receive PCP prophylaxis with TMPSMX for at least 6 months in general ${ }^{7,10)}$. Several studies have reported late-onset PCP which developed after 6 months of prophylaxis, or even after a year post-transplantation $^{5,11)}$. Our three cases also had late-onset PCP more than a year post-transplantation after the completion of routine PCP prophylaxis for 6 months, suggesting that transplantation recipients are not free from risks of PCP after a year and thereafter.

There are multiple risk factors contributed to PCP in renal transplant recipients; transmission in graft, recipient immunity, types and amounts of immunosuppressive drugs, occurrence of graft rejection, and previous or concomitant cytomegalovirus (CMV) infection ${ }^{8,9,12)}$. The major contributor to PCP occurrence is immunosuppressive therapy, although the effect of each individual immunosuppressant is unclear. Glucocorticoids suppress adaptive immunity and inflammatory response, which is a not only risk factor for PCP infection but also treatment of severe $\mathrm{PCP}^{13)}$. It is also well-known that MMF is a risk factor for $\mathrm{PCP}^{9)}$. Our patients maintained deflazacort or predinisolone and discontinued MMF temporarily for PCP treatment. Several studies showed that tacrolimus has the higher risk for PCP than cyclosporine and sirolimus also increased the risk of PCP in renal transplant recipients ${ }^{2,9)}$. All our three patients of late-onset PCP were taking tacrolimus with a mean trough level of 5-10 ng/mL, which was within target ranges of maintenance immunosuppression ${ }^{14)}$. Based on these knowledge, the third patient was expected to be high risk for PCP infection because of previous acute rejection, additional chemotherapy for PTLD, and sirolimus medication. Regarding CMV infection, all three patients had no CMV infection for the first 6 months after kidney transplantation.

Pneumonia is a major presentation of Pneumocystis jirovecii infection. PCP in organ transplant recipients manifest abrupt fever, dyspnea, and respiratory difficulty. Chest radiography in the patients reveals fine reticular interstitial change, suggesting atypical pneumonia ${ }^{15)}$. These non-specific symptoms and findings are common in PCP in renal transplant recipients. Patients in this study also had nonspecific abrupt symptoms like fever, sore throat, cough, dyspnea, and respiratory difficulty over the course of a few day, and showed diffuse reticular opacity in chest radiography.

Oral TMP-SMX is the first treatment of choice and prophylaxis for PCP. The recommend duration of treatment is at least 14 days but can be extended to 21 days in severe infection or when clinical symptoms are not improved ${ }^{13)}$. Depending on patient's condition, either oral or intravenous administration is possible because of excellent oral bioavailability. Clinical improvement is usually observed after 4-8 days of treatment. If patients show worse course or have severe adverse effects including elevated serum creatinine or potassium level, cytopenia, and gastrointestinal disorder, alternative agents like pentamidine, dapsone, and atovaquone should be considered ${ }^{15)}$. Our patients from the case all responded well to the initial treatment with intravenous TMP-SMX and received another additional 6-12 months of prophylaxis. However, our third patient who had two more episodes of PCP recurrence fulfilled more risk factors than the other two patients; she had experienced previous acute rejection and later received sirolimus medication and chemotherapy for PTLD which possibly explains the increased risk for PCP. Secondary prophylaxis for recurrent PCP in transplantation recipients are still under debate, and there is no definite consensus of duration. Recent study recommended that the high-risk patients such as those with enhanced immunosuppressive therapy and recurrent infection should receive longer or lifelong PCP prophylaxis ${ }^{5)}$.

The mortality rate of PCP in adult renal transplant recipients has gradually decreased from 33\% to about $14 \%$ recently ${ }^{3,16)}$. Early diagnosis, treatment, and the improved supportive care have contributed to this better outcome. So far, it is hard to assess the outcome of PCP in pediatric renal transplant patients due to limited data for children. However, this report showed that all three pediatric patients were rapidly diagnosed with PCR methods in few days, received treatment with intravenous TMP-SMX at least 3 weeks, and fully recovered. 
We report three cases of late-onset PCP more than a year after pediatric kidney transplantation all of whom completed 6 months of routine PCP prophylaxis. This study suggests that renal transplant patients have a lifelong risk of PCP manifesting as non-specific symptoms of pneumonia. Therefore, clinicians should bear in mind the possibility of opportunistic infections such as PCP in their patients.

\section{Patients consent}

This study was approved by the institutional review board (IRB), and the informed consents were waived due to the nature of the retrospective study [IRB number S2020-0313-0001].

\section{ORCID}

Soojin Hwang https://orcid.org/0000-0002-1779-237X

Jiwon Jung https://orcid.org/0000-0003-4333-3270

Joo Hoon Lee https://orcid.org/0000-0001-8010-3605

Young Seo Park https://orcid.org/0000-0001-7653-2036

\section{Conflicts of Interest and Source of Funding}

The authors declare that they have no potential conflicts of interest or funding source.

\section{References}

1. Fine RN, Martz K, Stablein D. What have 20 years of data from the North American Pediatric Renal Transplant Cooperative Study taught us about growth following renal transplantation in infants, children, and adolescents with end-stage renal disease? Pediatr Nephrol 2010;25:739-46.

2. Neff RT, Jindal RM, Yoo DY, Hurst FP, Agodoa LY, Abbott KC. Analysis of USRDS: incidence and risk factors for Pneumocystis jiroveci pneumonia. Transplantation 2009;88:135-41.

3. Arichi N, Kishikawa H, Mitsui Y, Kato T, Nishimura K, Tachikawa R, et al. Cluster outbreak of Pneumocystis pneumonia among kidney transplant patients within a single center. Transplant Proc 2009;41:170-2.

4. Rodriguez M, Fishman JA. Prevention of infection due to Pneumocystis spp. in human immunodeficiency virus-negative immunocompromised patients. Clin Microbiol Rev 2004;17:770-82, table of contents.

5. Perez-Ordono L, Hoyo I, Sanclemente G, Ricart MJ, Cofan F, PerezVilla F, et al. Late-onset Pneumocystis jiroveci i pneumonia in solid organ transplant recipients. Transpl Infect Dis 2014;16:324-8.

6. Transplantation EEGoR. European best practice guidelines for renal transplantation. Section IV: Long-term management of the transplant recipient. IV.2.2 Chronic graft dysfunction. Immunological factors (alloimmunity). Nephrol Dial Transplant 2002;17 Suppl 4:8-11.

7. Kasiske BL, Zeier MG, Chapman JR, Craig JC, Ekberg H, Garvey CA, et al. KDIGO clinical practice guideline for the care of kidney transplant recipients: a summary. Kidney Int 2010;77:299-311.

8. Radisic M, Lattes R, Chapman JF, del Carmen Rial M, Guardia O, Seu F, et al. Risk factors for Pneumocystis carinii pneumonia in kidney transplant recipients: a case-control study. Transpl Infect Dis 2003:5:84-93.

9. Eitner F, Hauser IA, Rettkowski O, Rath T, Lopau K, Pliquett RU, et al. Risk factors for Pneumocystis jiroveci pneumonia (PCP) in renal transplant recipients. Nephrol Dial Transplant 2011;26:2013-7.

10. Yazaki H, Goto N, Uchida K, Kobayashi T, Gatanaga H, Oka S. Outbreak of Pneumocystis jiroveci pneumonia in renal transplant recipients: $P$. jiroveci is contagious to the susceptible host. Transplantation 2009:88:380-5.

11. McCaughan JA, Courtney AE. Pneumocystis jiroveci pneumonia in renal transplantation: time to review our practice? Nephrol Dial Transplant 2012;27:13-5.

12. Neofytos D, Hirzel C, Boely E, Lecompte T, Khanna N, Mueller NJ, et al. Pneumocystis jiroveci i pneumonia in solid organ transplant recipients: a descriptive analysis for the Swiss Transplant Cohort. Transpl Infect Dis 2018;20:e12984.

13. Ewald H, Raatz H, Boscacci R, Furrer H, Bucher HC, Briel M. Adjunctive corticosteroids for Pneumocystis jiroveci pneumonia in patients with HIV infection. Cochrane Database Syst Rev 2015: CD006150.

14. Gaston RS. Maintenance immunosuppression in the renal transplant recipient: an overview. Am J Kidney Dis 2001;38(6 Suppl 6):S25-35.

15. Martin SI, Fishman JA, Practice ASTIDCo. Pneumocystis pneumonia in solid organ transplantation. Am J Transplant 2013;13 Suppl 4:272-9.

16. Iriart $X$, Bouar ML, Kamar N, Berry A. Pneumocystis Pneumonia in solid-organ transplant recipients. J Fungi (Basel) 2015;1:293-331. 